\title{
FORMAS DE HABITAR EL MUNDO RURAL ENTRE LOS JÓVENES EN MÉXICO
}

\author{
Luis Alberto Hernández de la Cruz ${ }^{1}$
}

\begin{abstract}
Resumen
Desde una perspectiva dinámica de los procesos socio-territoriales, observamos que lo rural se ha conformado a partir de la convergencia de múltiples actividades económicas y formas de vida. Partiendo del punto de vista de los actores sociales y especialmente de los jóvenes habitantes de dos municipios que conforman al Valle de Tehuacán, Puebla en la región centro de México, buscamos mostrar la diversidad y las incesantes transformaciones que estos territorios y sus pobladores han experimentado, contribuyendo a la construcción de nuevas formas de vivir y de imaginarse en el mundo rural, contribuyendo a la redefinición de los papales sociales y las formas de concebir estos territorios.
\end{abstract}

Palabras clave: Ruralidad, territorio, jóvenes, cultura

\section{MODOS DE VIVER ENTRE A JUVENTUDE RURAL NO MÉXICO}

\section{Resumo}

Nossa proposta centra-se entender o sistema rural como um sistema sócio complexo compreendendo uma materialidade e uma complexa relação entre a subjetividade dos habitantes e da estrutura socioeconômica. Este trabalho analisa, a diversidade e as constantes mudanças territorial e social do ponto de vista dos atores sociais, especialmente em jovens em dois municípios que compõem o Vale do Tehuacan, Puebla. Nesses municípios são vistas novas maneiras de viver e imaginar o mundo rural, mas também novos padrões de diferenciação entre as comunidades e a redefinição dos papéis social contribuindo para a construção de novas formas de imaginar, mas também novos padrões de diferenciação entre as comunidades e a redefinição dos papéis social.

Palavras-chave: Ruralidade, território, juventude, cultura

\section{INTRODUCCIÓN}

Para la primera mitad del siglo $X X$ la mayoría de los países latinoamericanos eran predominantemente agrarios, con centros urbanos que constituían ejes de actividad sobre los cuales giraba la vida económica, social y

1 Licenciatura en Sociología (Universidad Autónoma Metropolitana Unidad Iztapalapa). Maestro en Estudios Sociales (UAM-I). Doctor en Geografía (UNAM). Prof. Universidad Autónoma Metropolitana Unidad Cuajimalpa (UAM-C) Email: Indzdelacruz@yahoo.com 
cultural. Los sectores rurales estaban dedicados únicamente a la agricultura y ganadería, como actividades primarias con tendencia hacia la agroexportación y a la subsistencia familiar.

También lo rural estaba relacionado con imágenes que evocaban armonía y consenso, como un espacio apreciado, por estar relacionado con la tranquilidad y la comunión con la naturaleza. Un lugar para retirarse del ritmo cada vez más acelerado de la vida urbana y unirse con la vida comunitaria, o bien como un espacio en desaparición en donde paulatinamente es difícil distinguir sus particularidades de las pequeñas ciudades (ENTRENA, 1998).

Actualmente resulta difícil caracterizar al mundo rural de esta manera, diversos procesos económicos y sociales han confluido para entender que lo rural está conformado por diversas realidades que, difícilmente pueden definirse de manera dicotómica (MURDOCH Y PRATT, 1997; WOODS, 2009). Los primeros estudios de lo rural basaron sus investigaciones en criterios de comparación con lo urbano, obstaculizando el desarrollo de una reflexión que explicara sus particularidades desde la propia dinámica rural (REDFIELD, 1944; WIRTH, 1962).

Por otro lado, en lo que refiere a los estudios sobre los jóvenes en México la mayoría de las investigaciones se han realizado principalmente en ámbitos urbanos, buscando comprender las distintas configuraciones identitarias y prácticas juveniles de ciertos grupos (FEIXA, 1998) y también reflexiones que se centraron en el análisis global de la juventud, abordándose temas demográficos, educativos, laborales, migratorios, de salud, drogadicción y adicciones, participación política, género, violencia, religión y valores juveniles (GUZMÁN, 1994; NAVARRETE, 1992; STERN, 1998).

En este sentido, parece ser que la idea predominante sobre las comunidades rurales es que prácticamente los grupos juveniles eran inexistentes. Sin embargo, en la actualidad podemos observar que existen territorios rurales en donde los jóvenes debido a los cambios demográficos, sociales y culturales son actores importantes dentro de sus comunidades de ahí la importancia de conocer un poco más del mosaico generacional que se está construyendo dentro de estos espacios tan diversos.

Hoy las fronteras entre lo urbano y lo rural son menos claras, ya sea por el crecimiento urbano o por los múltiples desplazamientos laborales y sociales, que han sobrepuesto ambos espacios. En este contexto, lo rural se constituye en un espacio de análisis y reflexión muy importante debido a las transformaciones que experimenta. En ese sentido, el presente artículo se basa en una investigación que analizó las transformaciones socio-territoriales en el contexto de la reestructuración económica, desde el punto de vista rural.

El objetivo principal de este trabajo es conocer algunos elementos que inciden en las formas de habitar el mundo rural entre los jóvenes de dos municipios del Valle de Tehuacán, Altepexi y Ajalpan. A partir de una perspectiva constructivista analizamos la forma como sujetos y territorio se relacionan y caracterizan algunas particularidades del mundo rural contemporáneo.

En el trabajo de campo se realizaron 24 entrevistas a los pobladores de los municipios mencionados de las cuales 10 correspondieron a jóvenes de entre 15 y 29 años. Con base en la información de estas entrevistas en específico intentamos identificar los cambios y acontecimientos más importantes en la vida de los habitantes, así fueron los propios habitantes quienes señalaron los factores que creían más relevantes y dieron cuenta de los hechos trascendentes que han transformado tanto su territorio como a ellos mismos. 
En primer lugar presentaremos nuestro enfoque de análisis, el cual se caracteriza por articular los elementos subjetivos y los estructurales del territorio para constituir una perspectiva constructivista de los procesos rurales en donde el punto de partida y eje más importante es la subjetividad de los actores, posteriormente reconstruiremos la historia de los municipios de estudio, para después centrarnos en el análisis de las nuevas formas de habitar lo rural entre los jóvenes y finalmente presentar algunas reflexiones finales.

\section{EL CONSTRUCTIVISMO GEOGRÁFICO RURAL}

La propuesta de análisis de este trabajo considera que la interrelación entre estructuras sociales y subjetividades colectivas e individuales genera la construcción social del espacio. En el conocimiento de las formas en la que los sujetos interrelacionan los elementos objetivos que configuran su interpretación de su territorio, buscamos entender las formas subjetivas a través de las cuales interpretan la realidad. De tal manera que los sujetos significan y constituyen su realidad a partir de las interacciones entre ellos y el medio que les rodea. En donde la dimensión socio-histórica incide en la manera en cómo se configuran las características básicas de los territorios. El territorio además de ser un espacio que ha sido valorizado instrumentalmente (bajo el aspecto ecológico, económico o geopolítico), también lo es culturalmente. En el proceso de valorización la interacción social diaria entre los habitantes permite que el territorio sea, significado, transformado y vivido.

A mediados de los años setenta LEFEBVRE (1991) colocó las bases de la perspectiva humanista en la geografía, tomando como punto de partida el materialismo histórico y la dialéctica hegeliana buscó desarrollar una postura que superará a los dogmas del marxismo ortodoxo y las presuposiciones de los análisis funcionalistas. Algunos de sus postulados influyeron directamente al desarrollo de una perspectiva constructivista de geográfos como Di Meo y Raffestin.

Lefebvre, debatió con las concepciones duales, que definían al espacio como, lo concreto, lo material y por otra parte con aquellas que lo definían como producto de las representaciones cognoscitivas de los seres humanos. Para ello elaboró una propuesta trialéctica del espacio social, constituida por el espacio percibido, el espacio concebido y el espacio vivido. El primero se encuentra relacionado con la práctica social materializada, el segundo es definido por las representaciones del espacio mientras que el tercero es producto de la relación espacialidad-historicidad-sociedad. El punto de partida de esta propuesta es el concepto de producción del espacio, que permite superar la oposición sujeto-objeto.

Durante los años ochenta el concepto de territorio se presenta como una categoría constituida por la interrelación de diferentes elementos históricos, culturales, sociales. Para Raffestin (1980) y Di Méo (1991) en términos generales el territorio comenzó a entenderse como un entorno en el que convergen lo económico, lo ideológico, lo político y lo social.

Raffestin en su obra más relevante Pour une géographie du pouvoir (1980), define al espacio como la prisión original, mientras que el territorio es la prisión que los hombres se dan a sí mismos. Para este autor los procesos de organización territorial deben analizarse en dos niveles distintos, pero en constante interacción: el de la acción de las sociedades sobre los soportes materiales de su existencia, y el de los sistemas de representación. Desde esta perspectiva el territorio se entiende como la manifestación espacial del poder fundamentado en relaciones sociales, 
relaciones determinadas, en diferentes grados, por la presencia de energía acciones y estructuras concretas - y de información -acciones y estructuras simbólicas.

Por otra parte, Di Meo (1991) quien ha realizado la propuesta teórica más relacionada con la perspectiva constructivista, cimienta su reflexión en la articulación de tres ejes las personas, la sociedad y el espacio. La convergencia de estos elementos es dinámica, el espacio influye en los individuos y los individuos asimismo lo moldean. También plantea la misma articulación entre el individuo y la sociedad, en ese juego de relaciones recíprocas constantemente destaca la importancia de lo subjetivo junto a lo objetivo, de lo material y lo no material, de lo individual, lo social y lo espacial. El territorio confiere a la realidad social una coherencia material basada en signos y símbolos incorporados en objetos, lugares y paisajes. El territorio funciona entonces como una mediación socio-espacial que puede facilitar la creación de una identidad (esquema 1).

Esquema 1. El territorio como un sistema complejo

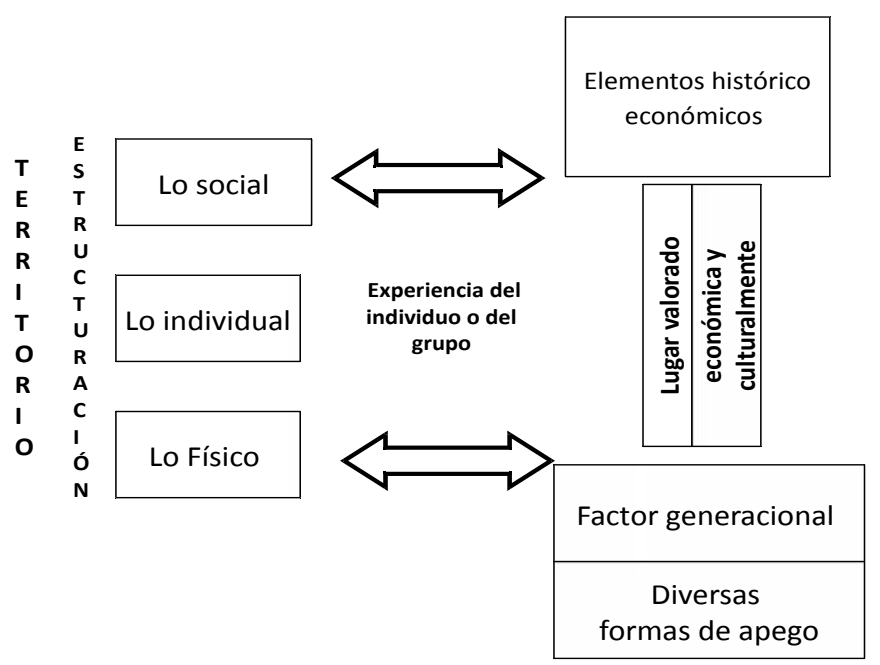

Fuente: Elaboración propia

El territorio sería entonces el resultado de la apropiación y valorización del espacio; en el primer caso enfatiza la relación utilitaria del espacio (por ejemplo, en términos de explotación económica o de ventajas geopolíticas), mientras que en el segundo se destaca el papel del territorio como espacio de sedimentación simbólicocultural, como objeto de inversiones estético-afectivas o como soporte de identidades individuales y colectivas (GIMÉNEZ, 2000). Desde esta perspectiva el territorio rural es, a la vez, un centro de significado, es el contexto externo de las acciones y a través de los testimonios orales materializamos el significado de las experiencias de vida, así el territorio, es un espacio vital, el espacio de la experiencia cotidiana, el espacio de la experiencia histórica, un ámbito de identidad del grupo humano que la habita.

En la apropiación del territorio rural interactúan las diversas formas en que los sujetos influyen en su constitución, y los elementos en los que el territorio incide 
sobre la sociedad y el sujeto. De esta forma el territorio rural es portador de visiones, historias y aspiraciones de quienes viven y lo conforman. El territorio rural es por lo tanto, un producto de la capacidad que tienen los hombres para transformar con su trabajo la naturaleza que les rodea y también sus propias relaciones sociales. A través de él se reconoce la imbricación histórica de la relación naturaleza y cultura.

El territorio rural es un espacio que se encuentra en una continua transformación cultural y económica. Las transformaciones de los procesos de trabajo y producción modifican las formas de socialización, las instituciones sociales, los patrones, valores socioculturales y los modos de vida. Lo rural es una dimensión de lo social, una construcción, en la que convergen tiempo e historia. Esto implica colocar en el centro de la reflexión a los sujetos y partir del supuesto de que los territorios rurales -al igual que todos los procesos sociales- están en constante transformación por lo que existe una relación intrínseca entre historia-sujetos y cambio.

\section{JÓVENES Y RURALIDAD EN MÉXICO}

En México, la mayoría de las investigaciones sobre los jóvenes se han realizado desde y en la ciudad, buscando comprender en términos generales cómo las prácticas juveniles de ciertos grupos se materializan en un tipo de identidad sociocultural y también reflexiones de carácter más estructural centradas en el análisis global de la juventud, abordándose temas sociales, demográficos, educativos, laborales y migratorios. A este respecto REGUILLO (2010) menciona que el debate en torno a los jóvenes en el país se ha centrado en la dimensión estudiantil y en esta medida, los jóvenes varones y urbanos se convirtieron en los depositarios de los significados de "ser joven".

Durston (1996) señala que la juventud rural está empezando a perder su invisibilidad en la discusión sobre el futuro del mundo rural en América Latina. Sociólogos, planificadores y políticos señalan su papel crucial en las transformaciones en marcha en la agricultura y en las demás facetas de la vida en el campo. Sin embargo, es necesario conocer las realidades diversas que viven las diferentes juventudes rurales que existen al interior de cada país, de cada región, para que los datos sólidos que en algún momento llegan a construir estereotipos de los jóvenes rurales sean reinterpretados a la luz de las subjetividades particulares.

Recientemente debido a los efectos de múltiples transformaciones y procesos particulares que han ocurrido en los territorios rurales se ha vuelto la mirada a ese sector que en algún momento se consideró ajeno al fenómeno de la(s) juventud(es). De esta manera las y los jóvenes rurales aparecen como sujetos protagónicos relacionados con dinámicas sociales y económicas diversas. Por lo tanto, surge la necesidad de indagar sobre estas realidades revitalizadas a la luz de la carencia de investigaciones que aborden estas particularidades.

Algunas investigaciones que se ubican en esta perspectiva dan cuenta desde aspectos que tienen que ver con la conceptualización de la juventud rural (BEVILAQUA, 2009), hasta otras que abordan las principales situaciones problemáticas a las que se enfrenta este grupo poblacional: pobreza, aislamiento, violencia, marginación, discriminación ética y falta de equidad en el acceso a ciertos satisfactores (BONFIL, 2001, PACHECO, 2010).

Sin embargo, este tipo de reflexiones se centran más en los elementos estructurales por lo que consideramos muy importante conocer desde el punto de 
vista de los jóvenes rurales sus preocupaciones, problemáticas y las formas de concebir su territorio para que del mosaico de estas reflexiones particulares vayan construyendo una visión global de un fenómeno tan heterogéneo.

En este sentido, con frecuencia el término "juventud" se utiliza genéricamente, asociándose a grupos de edad particulares o a una etapa específica del ciclo vital que presenta características comunes en todas las poblaciones. En realidad, la juventud de un territorio, un país o una región se compone de sectores y grupos heterogéneos, con condiciones de vida desiguales y con diversas formas de apropiación del medio natural, cultural y social. Así, por ejemplo, ser joven, y ser un joven del medio rural, es una condición particular que no viven igual jóvenes rurales de la misma región, inclusive de un mismo país (IICA, 2000).

Por otro lado, una categoría de análisis social que permitiría conocer más las particularidades de estas realidades, es el género, ya que uno de los estructuradores más poderosos de la juventud es el género (URTEAGA, 2010). En lo que refiere a este concepto desde el resurgimiento de la reflexión feminista a finales de los años sesenta, se revela la asimetría social entre hombres y mujeres, el género emerge como una categoría trascendental para el análisis de lo social y se comprende la diferencia entre género y sexo. Así, el primero se concibió como una creación social y cultural que se desprende del segundo. Estas proposiciones colocan sobre la mesa de estudio de las ciencias sociales, las divisiones de género como un eje fundamental de la diferenciación de lo social, oponiéndose a la idea que se asume con frecuencia que el género es solo un atributo de la feminidad. En este sentido se reconoce que las relaciones de género son básicamente relaciones de poder, desiguales y jerárquicas y no meras dicotomías o relaciones simétricas y complementarias.

En este sentido y relacionado con las nuevas realidades en los espacios rurales tiempo atrás parecía que las mujeres estaban confinadas al ámbito reproductivo. Sin embargo, comenzamos a observar las transformaciones que se han acarreado al respecto y un claro ejemplo es lo que sucede en nuestros territorios de estudio que señalaremos más adelante. Es por ello que consideramos importante problematizar e indagar sobre la relación juventud- género-ruralidades.

Así el territorio rural puede ser entendido como un sistema dinámico, como una expresión socioespacial, como un sistema sociocultural que tiene rasgos particulares, pero al mismo tiempo responde a transformaciones de otra escala. La gran diversidad de cambios en el mundo rural es una muestra de la imposibilidad de seguir pensándolo como un espacio hermético. Así lo rural no es solo el territorio sobre el cual la ciudad se ha reproducido, también es producto de la articulación entre las relaciones económicas y las formas en las que los individuos lo perciben, imaginan y valoran de modos diversos.

En el análisis y estudio del territorio rural es importante observar las interacciones entre las diferentes dimensiones que lo constituyen, pero con especial énfasis en la estructuración de lo social y de lo individual, frente al entorno físico. En este proceso es importante valorizar la experiencia del individuo o del grupo, buscando comprender el comportamiento y las formas de sentir de las personas en relación con sus territorios de residencia. Además también incorporar los elementos histórico-económicos como una parte importante que conforma las formas de valorización de territorio. Es decir, cada individuo y grupo humano, genera una visión del mundo que se expresa a través de sus actitudes y valores.

En términos generales esta propuesta se basa en un constructivismo que parte del análisis de los procesos de interacción entre las estructuras sociales y las subjetividades individuales y colectivas que permiten a los sujetos constituir 14 
significados y pertenencias sociales y territoriales. Un constructivismo para el que cada expresión individual esta mediada por la realidad, que es significada a partir de los elementos culturales compartidos por los sujetos, materializados en sus prácticas cotidianas.

\section{EL VALLE DE TEHUACÁN, PUEBLA}

Tehuacán está situado al sureste del estado de Puebla, históricamente, es un territorio que permite enriquecer el conocimiento sobre los primeros pobladores de la región. Además es una referencia básica para profundizar en el conocimiento sobre la historia de la agricultura, ya que se encontraron ejemplares de mazorcas de maíz que muestran la domesticación de esta planta, desde su estado silvestre hasta alcanzar el tamaño y las variedades

que conocemos en la actualidad.

En lo que refiere a los municipios que en su momento permitieron conformar al Valle, resaltan por sus particularidades, Altepexi y San Juan Bautista Axalpan (Ajalpan), fueron los primeros territorios en donde se albergaron núcleos fijos de población que se dedicaron a la agricultura. La interconexión en el sistema de riego, la producción y comercialización entre Altepexi y Ajalpan permitió que se erigieran en municipios que a principios de siglo $X X$ desarrollaran también actividades económicas alternas al quehacer agrícola. En Ajalpan se elaborarían ladrillo y tejas de manera artesanal, mientras que en Altepexi se instalaría una fábrica de telas. Posteriormente en estos municipios, el trabajo industrial, materializado en la maquila de confección, sería el más importante.

\section{Mapa 1 - Ubicación de la zona de estudio}

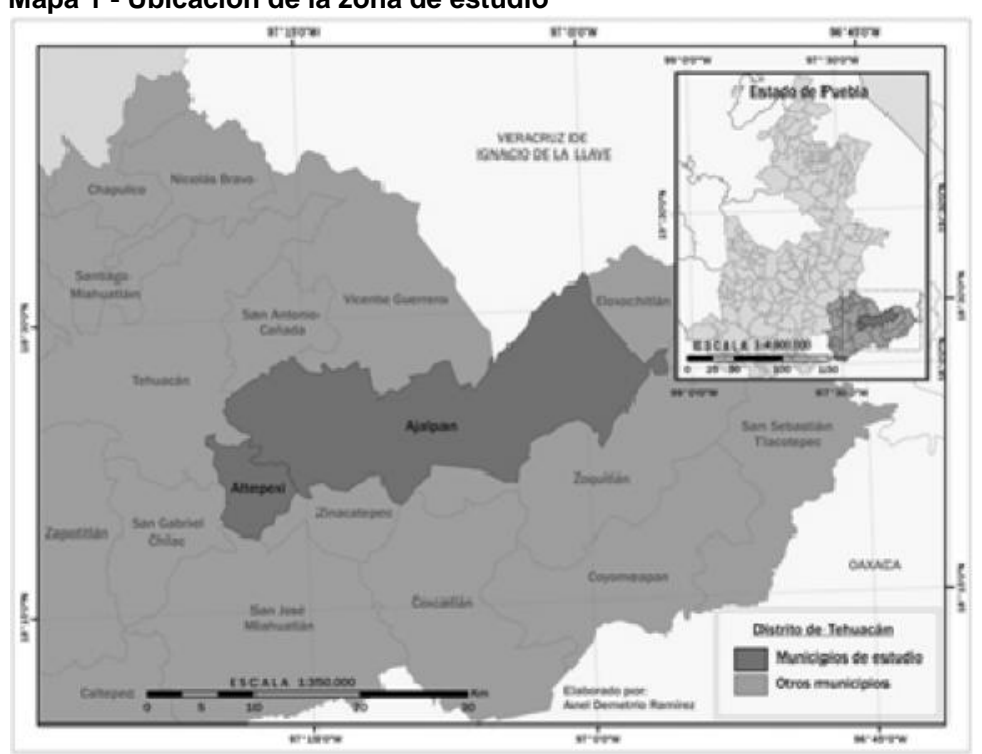

Fuente: Elaboración por Anel Demetrio 
La instalación de las primeras maquiladoras se da entre 1971 y 1973; inicialmente se dedicaron a confeccionar los uniformes de las industrias más importantes hasta ese momento, las embotelladoras y las granjas avícolas. También vendían sus productos en los principales tianguis de ropa del Estado de Puebla. A partir de 1994, con el Tratado de Libre Comercio de América del Norte, la región se tornó en un enclave para los mercados nacional e internacional. El auge de la maquila en toda la región fue impulsado en primer lugar por la crisis del campo, el declive de las industrias refresqueras y avícolas y la apertura comercial. En este contexto los productores locales lograron vincularse con algunas marcas mundiales como Levis, Gap, Tommy, Mossimo, etc.

Este apogeo contribuyó a la migración intra-regional, con la llegada de trabajadores de los municipios aledaños y de la zona de la sierra poblana quienes alimentaron por mucho tiempo a la maquila, conformando asentamientos irregulares y nuevas colonias las cuales contribuyeron al crecimiento de la ciudad de Tehuacán (BARRIOS y SANTIAGO, 2004).

Hoy, es posible ver maquiladoras en municipios en los que tradicionalmente no se encontraban localizadas. Este fenómeno fue impulsado por las estrategias que el gobierno estatal realizó para remediar la crisis del sector agrícola, además de convertirse en un instrumento para aminorar los procesos migratorios tan importantes en la región. Las maquiladoras de confección se convirtieron en la principal fuente de ingresos para muchos habitantes del Valle.

\section{5. (RE)CONOCIENDO A LOS JÓVENES RURALES}

Tanto Ajalpan como Altepexi con territorios rurales se van conformando en espacios estratégicos en la reproducción económica de la región. Esta nueva dinámica genera una diversificación en el uso y apropiación del territorio rural: por un lado, se conforman espacios en donde la producción se destina a lo agrícola; y, por otro lado, espacios en donde se realiza alguna actividad ligada a la producción industrial, pero al mismo tiempo pueden elaborar algún tipo de artesanía.

Son territorios rurales que a lo largo de su historia han estado relacionados con algún tipo de labor manufacturera, por lo tanto aprender las diversas tareas que entrañan los nuevos trabajos es menos dificultoso para la población. Los pobladores han combinado distintos quehaceres laborales y modos de vida a lo largo de su historia. Estas características cuestionan de manera directa las formas en las cuales se había entendido a los habitantes de estos territorios, por ejemplo se pensaba que estaban insertos completamente en una sociedad tradicional, donde los contenidos culturales y los valores se trasmitían en forma verbal. Por el contrario los territorios rurales que investigamos muestran otro tipo de realidad, son un ejemplo de la diversidad de elementos que los conforman, por lo que es importante conocer cuáles son estos componentes, cómo están relacionados y de qué manera son comprendidos por los habitantes.

Los jóvenes entrevistados tienen edades que van de los 15 a los 29 años, los más jóvenes viven en Altepexi mientras que los mayores son residentes de Ajalpan (cuadro 1). Son municipios vecinos por lo que usualmente quienes viven en Ajalpan tienen familiares o amigos en Altepexi y viceversa, también en algún momento el municipio de Altepexi formó parte de Ajalpan, además de compartir un pozo de agua que años atrás se secó.

Hay historias diversas entre estos jóvenes, destacan aquellas en las que el trabajo y la escuela son dos actividades que realizan diariamente, otras en las cuales el trabajo en la maquila es el más importante y la escuela se ha dejado. 
Por ejemplo en el caso de Miguel y Roberto de Ajalpan tenemos que el primero aún sigue estudiando y por las tardes o los fines de semana se dedica a ayudar a la familia en la elaboración de cestería de carrizo que es una de las actividades artesanales más importantes de este municipio, mientras que en el caso de Roberto trabaja diariamente en la maquila a pesar de que en algún momento también se dedicara a la elaboración de canastas, sin embargo, debido a que en ocasiones esta actividad no es tan solicitada. Mientras que la maquila de confección tanto las empresas medianas como los talleres siempre solicitan mano de obra.

Cuadro 1 - Distribución por edades según municipio

\begin{tabular}{|l|r|}
\hline AJALPAN & ALTEPEXI \\
\hline Miguel 15 años & José 15 años \\
\hline Roberto 18 años & Arturo 15 años \\
\hline Alberto 21 años & Jesús 19 años \\
\hline Noemí 24 años & Diego 20 años \\
\hline Ana 29 años & Edgar 21 años \\
\hline
\end{tabular}

Fuente: Elaboración propia

En el caso de Jorge y Arturo de Altepexi ambos de 15 años, uno de los primeros recuerdos que comparten sobre las actividades laborales en las que se empleaban sus padres es la maquila, para ellos el campo ha dejado de ser una opción a pesar de que sus abuelos aún se dedican a esta actividad "porque antes los de nuestra edad estaban acostumbrados para ir al campo y nosotros ya no, ya mejor se van a las maquilas o a estudiar".

A diferencia de otros municipios para estos dos jóvenes Altepexi es un espacio muy tranquilo donde no hay mucho ruido, en el cual puedes pasear sin ningún problema con tus amigos, ellos son en este momento una de las principales razones por las que les gusta vivir en su municipio, además que en los otros hay más gente y ruido.

En el caso de las mujeres Noemi de Ajalpan tuvo que dejar la prepa para poder trabajar, actualmente labora en la biblioteca del municipio, recuerda que cuando era estudiante durante el periodo de vacaciones trabajó en la maquila para poder ayudar a su mamá y abuela y poder continuar en la escuela. Sin embargo, se embarazo eso implicó dejar también la escuela, aunque vive en unión libre (su pareja también dejo el bachillerato para trabajar en la maquila) tiene que trabajar para poder completar para los gastos diarios. En el futuro ella espera dedicarse también a la maquila pero como socia ya que su pareja junto con unos primos están pensando montar un pequeño taller.

Por otro lado, Ana también habitante de Ajalpan recuerda su infancia muy tranquila, sencilla, hasta cierto punto para ella muy pobre, debido en parte a que las fuentes de trabajo más importantes cuando era niña eran la cestería de carrizo, la elaboración de tejas de barro y pequeñas fabricabas de tabique. Ella al igual que la mayoría de los jóvenes de la región comenzó a trabajar a los 13 años, recién termino el 1 año de secundaria y la presión de su papá para que dejara de estudiar debido a los gastos que esto implicaba, por eso decidió emplearse para poder continuar en la 
escuela, empezó a laborar en la maquila durante las vacaciones y después todos los días por las tardes.

Esto implicó enfrentarse a su papá ya que en sus propias palabras "es un hombre que era enemigo de que las mujeres fueran a trabajar, de hecho él me dijo que si iba a trabajar que él no quería que algún centavo fuera para la casa o para los hermanos, sino que lo ocupara para la escuela".

De esta manera y con el apoyo de sus maestros pudo llegar hasta la preparatoria pero se convirtió en una actividad muy pesada ya que para ese momento ya no podía combinar escuela y trabajo por lo que tuvo que dejar la escuela y dedicarse por completo a laborar.

En el caso de Jesús de Altepexi que forma parte de la banda municipal su principal referente es el campo debido a que sus padres y abuelos en su mayoría se dedicaban a la siembra de jitomate y la milpa, por un tiempo también él se dedicó a esta actividad y esto le ha permitido hasta el momento poder seguir estudiando aunque su sueño es tener una banda propia ya que en este negocio se gana un poco más de lo que gana un campesino "por ejemplo un campesino en todo el día por sus ocho horas gana 120 pesos y en una tocada lo ganas en una hora". Por lo que su relación con el campo a temprana edad le ha permitido conocer algunas tradiciones que considera son importantes para su localidad.

Alberto por otro lado de Ajalpan su experiencia en el trabajo de la maquila le ha permitido darse cuenta rápidamente como empieza a desaparecer y reconocer que antes abundaba el trabajo y la paga era buena sin embargo actualmente ya no es así "ya no pagan horas extras, ya casi se trabaja gratis a veces nos explotan"

El arribo de nuevas formas de trabajo, comenzó a transformar el tejido social, los jóvenes ya no quieren seguir la vida de aquellos padres que tienen tierras: sembrar, labrar, regar y cosechar. Hoy la producción en algunos casos es exclusivamente para el autoconsumo, muy pocos sectores, aquellos que cuentan con mayor número de tierras y recursos hidráulicos son los que han logrado mantenerse.

También se han conformado pandillas que en los bailes públicos o fiestas en ocasiones riñen. Ahora es común observar en Ajalpan y Altepexi, una gran cantidad de jóvenes con características de las llamadas tribus urbanas (cholos, emos, skatos). El consumo de alcohol y el uso de drogas como la marihuana, la cocaína y el cemento, se han convertido en prácticas comunes. En varias entrevistas se señala que en Altepexi esta situación es un verdadero problema para los vecinos y las autoridades, mientras que en Ajalpan es una situación que comienza a ser más visible. "Aquí hay un dicho en Altepexi, que dice que si levantas una piedra o sale un borracho o un músico. A parte de abundar los alcohólicos, también hay muchos músicos" Jesús 19 años

La maquiladora de confección es la industria que modificó de manera más rápida económica y socialmente a los municipios de estudio. En las entrevistas realizadas las personas de mayor edad explican qué quienes han sido más afectados, son los jóvenes, tanto mujeres como hombres. Señalan que los jóvenes al observar que el trabajo en la maquila provee de un salario semanal fijo, prefieran emplearse en ella. La escuela también pasa a segundo plano. Por otro lado la gran mayoría ha dejado de hablar la lengua de los abuelos (el náhuatl), con estos cambios los padres y abuelos están alarmados ante esta súbita transformación.

En estas ruralidades, coexisten empresas que llegan a maquilar a grupos transnacionales, mundos rurales heterogéneos con campesinos, productores medios, grupos étnicos y nuevos desocupados. El mundo rural observado a través de estos dos municipios remite no solo a los vínculos histórico-económicos, también 18 
implica tomar en cuenta las transformaciones ligadas a una pluralidad de actividades, que modifican las formas de concebir al espacio más próximo, materializado en el territorio y en las relaciones sociales. Esto implica poner más atención en toda la red de transformaciones, pero siempre colocando las vivencias como eje de articulación a los pobladores, ya que ellos son los que experimentan en primera instancia los cambios.

En las calles se observa la articulación y contraposición de distintos modos de vida, algunas casas todavía siguen siendo de carrizo, revestidas con paredes de adobe, otras son de loza de concreto y algunas alcanzan los dos pisos. Trazos, que son menester de todos los días en las ciudades, se comienzan a observar en las paredes, grafitis de múltiples colores que contrastan con las canastas multicolores de las abuelas que van al mercado para comprar el mandado. Los más ancianos, en su mayoría, todavía regatean en su lengua materna los precios en el mercado, sus hijos viajan en bici cargando en la llanta trasera a los más pequeños de sus nietos, los jóvenes miran con atención las nuevas películas de moda, mientras que otros entablan conversaciones cibernéticas.

En este proceso de significación resaltan tres vínculos: el territorio, la familia y el trabajo. En gran parte de las entrevistas realizadas las personas entre 50 y 60 años relatan algunos de los cambios que consideran han ocurrido en su municipios los últimos diez años, entre los que sobresalen las formas de vestir y actuar de los pobladores. Señalan como una característica común de la manera de vestir de los pobladores dos décadas atrás fue el "vestido blanco" o mejor dicho la ropa de manta, que era la forma tradicional de vestir de algunos pueblos indígenas de la región, la cual era utilizada con orgullo por ser un elemento compartido por gran parte de la población.

En el discurso las comunidades se presentan como unidas y estables. Sin embargo, a partir del análisis de la historia común y particular de cada municipio el conflicto y el poder, son también elementos que inciden en las formas de apropiación de los habitantes de su entorno.

En gran parte de las entrevistas destaca un discurso sobre la división entre el indígena y el mestizo, el cual se reprodujo en la división territorial de cada municipio y que incide en las relaciones sociales establecidas entre los habitantes. En el caso de Ajalpan en varias entrevistas se enfatiza la distinción entre la gente de razón y del pueblo. Una habitante de Ajalpan señala que "la gente del pueblo se dedica a la canasta, hablan en náhuatl y la gente de razón no, tampoco usan trajes típicos y somos más claros"

Este fenómeno ha dado lugar a una configuración territorial muy particular “...la gente indígena o del pueblo esta confinada al oriente, los hacendados por la parte norte, en el centro habita la clase media" El municipio de Ajalpan basó su diferenciación y división territorial a partir de la negación de su pasado indígena. Aunque con matices, la gente de mayor edad aun reconoce la importancia que tiene, sin embargo son los jóvenes quienes consideran no tan importante este aspecto y han constituido sus propias formas de diferenciación tanto al interior y al exterior del municipio. Uno de los elementos que consideran todos los pobladores como signo de distinción entre los municipios es la "forma de hablar y de vestir, allá como hablan más náhuatl que aquí, su español es diferente, chistoso..."

En el caso de Altepexi observamos que esta división es totalmente opuesta, la tierra en este municipio es un recurso escaso y concentrado en pocas manos. Es importante señalar que existe una correlación directa entre quienes tienen tierra y 
quienes pueden explotar los depósitos de agua, que alimentan al municipio. Esto generó una diferenciación al interior del municipio de Altepexi, quien vive en el centro es considerado nativo y tiene derecho a poseer un mayor control sobre la tierra. En algunos casos también tiene mayor poder político y económico, mientras que los que viven a las afueras son los fuereños que no tienen acceso a la tierra ni derecho a alcanzar algún puesto político.

La tierra para los pobladores de mayor edad que tuvieron o tienen derecho a su uso, adquiere un significado muy distinto en comparación con quienes han tenido un acceso limitado o nulo. Los primeros han establecido vínculos económicos y afectivos más estrechos con su lugar de origen. Para quienes no han tenido acceso a la tierra, las relaciones sociales y las vivencias son los primeros elementos que permiten significar a su territorio. Así, la tierra que en principio es un bien económico, también se constituye en un bien simbólico que se relaciona con una historia particular. En Ajalpan el conflicto por la posesión de la tierra no es tan evidente, la tierra y el ser campesino, es percibido como una actividad residual hasta para quienes tienen acceso a ella y para poder obtener más beneficios económicos realizan otras actividades.

\section{CONSIDERACIONES FINALES}

La incorporación de elementos culturales de tipo urbano en la vestimenta de los pobladores jóvenes marca una diferencia entre la gente de ciudad y la gente de campo. Los jóvenes viven en el campo pero su estilo de vida es urbano.

Los roles sociales se transforman y adquieren otros sentidos, por ejemplo, las mujeres jóvenes tienen ahora acceso a los estudios y al trabajo; el ser mujer ya no sólo está relacionado únicamente con ser ama de casa o madre. Una de las características que distinguen a estos territorios rurales es que la pluriactividad que los configuró permite que algunas mujeres trabajen desde edades muy tempranas en comercios que se instalaron con la llegada de las empresas avícolas.

Para algunos abuelos la lengua y el cultivo del maíz son prácticas que afirman su identidad, para los jóvenes de los municipios la agricultura ha dejado de tener sentido, así como la lengua y la reivindicación del campesino. Esto no significa que menosprecien la vida de sus abuelos o padres, pero su percepción sobre lo rural está influida por una historia en la que el trabajo en el campo es considerado una actividad desvalorizada, mientras que la maquila de confección es para muchos un trabajo con más beneficios y con un estatus diferente. Sin embargo, esto no anula su pertenencia al mundo rural sino que se plantea en otro sentido. Estas formas de relacionarse con el territorio son reflejo de la historia que cada generación ha vivido.

En el caso de los abuelos y su relación más estrecha con la tierra está vinculada con los discursos que en su momento permeaban al campo mexicano, el cual era considerado el motor del desarrollo y quienes laboraban en él eran pobladores comprometidos con el desarrollo de la nación, esto implicaba ser reconocidos como un sector de la sociedad muy importante, que se distanciaba también de la imagen del indígena, remplazada por el campesino productor. Por otro parte, los hijos de estos campesinos trataron de mantener este discurso, sin embargo, las condiciones económicas y sociales eran muy diferentes a las de sus padres. El ideal de que la vida rural era una muestra del desarrollo no correspondía con la realidad inmediata. Para esta generación, esto implicó una recomposición en los elementos de apego hacia el territorio, mientras que para sus padres la tierra y el pueblo eran básicos para vivir en su municipio, para ellos la familia se constituye 
como el elemento principal que los une al territorio. En segundo lugar prevalece el trabajo, pero ahora se caracteriza por la transición hacia el empleo en diversas actividades, buscando en primer lugar el bienestar familiar. Para ellos, el campo es un lugar adecuado para vivir, ya que es tranquilo y no existen los mismos problemas de inseguridad que en la ciudad.

En las historias de los jóvenes observamos que el principal vínculo con el territorio es a través del trabajo en la maquila, varios de los entrevistados consideran que es el empleo que les permite acceder a un nivel de vida mejor que el de sus padres o abuelos. El salario y los bienes que consumen les permiten distanciarse del estigma de indígena y campesino que ha marcado su historia. A diferencia de sus abuelos y padres consideran que el campo es un lugar aburrido, en ocasiones atrasado, a diferencia de la ciudad en donde existen espacios de diversión. De igual manera el trabajo en el campo en comparación con el de la maquila es percibido como una característica del pasado que mantuvo a sus abuelos y padres alejados de ciertos bienes y servicios que actualmente son considerados necesarios para diferenciarse de otros habitantes.

Hablar de lo rural en la actualidad, por lo tanto, implica referirse a múltiples significados que se elaboran de manera individual y colectiva. Así el territorio es el espacio de resguardo de la familia, es el espacio de trabajo. Es también el espacio apropiado y con ello se constituye en un soporte y recurso básico, además un ámbito de vida, que es significado en la memoria personal y colectiva.

\section{BIBLIOGRAFÍA}

BARRIOS, M. y HERNÁNDEZ, R. Tehuacán: del calzón de manta a los blue jeans, Toronto: Comisión de Derechos Humanos y Laborales del Valle de TehuacánRed de Solidaridad de la Maquila, 2004.

BEVILAQUA, J. Juventud rural: Una invención del capitalismo industrial, Estudios Sociológicos, vol. XXVII, núm. 80. México: El Colegio de México, 2009.

BONFIL, P. ¿Estudiar para qué? Mercados de trabajo y opciones de bienestar para los jóvenes del medio rural. La educación como desventaja acumulada, Pieck, E. (coord.), Los jóvenes y el trabajo. La educación frente a la exclusión social. México: UIA/IMJ/ UNICEF, 2001

DI MEO, G. Géographies tranquilles du quotidien. Une analyse de la contribution des sciences sociales et de la géographie à l'étude des pratiques spatiales Cahiers de Géographie du Québec, Volume 43, n 118, avril, pp. 75- 93, 1999.

DURSTON, J. Juventud rural en América Latina: Reduciendo la invisibilidad", Padilla, J. (coord.), La construcción de lo juvenil. Reunión Nacional de Investigadores sobre Juventud. México: Causa Joven,1998

ENTRENA, F. Cambios en la construcción social de lo rural. De la autarquía a la globalización, Colección "Ciencias Sociales", Madrid: Técnos. 1998

FEIXA, C. El reloj de arena. Culturas juveniles en México. México: Causa Joven, 1998 
GIMENEZ, G. Territorio, cultura e identidades. La región sociocultural Rosales R. (coord.) Globalización y regiones en México. UNAM/ Miguel Ángel Porrúa. México pp 19-52, 2000.

GUZMÁN, C. (1994). Entre el deseo y la oportunidad: Estudiantes de la UNAM frente al mercado de trabajo. Cuernavaca: UNAM-Centro de Investigaciones Multidisciplinarias.

IICA (Instituto Interamericano de Cooperación para la Agricultura) (2000). Jóvenes y nueva ruralidad: Protagonistas actuales y potenciales del cambio Un acercamiento conceptual a la situación y a la importancia del desarrollo humano de los sectores juveniles de América Latina y el Caribe en la aurora del 2000. Serie Documentos Conceptuales No. 2000-02 Julio

LEFEBVRE, $H$. The production of space. Blackwell published/ Oxford \& Cambridge, 1991

MURDOCH, J. y PRATT, A. "From the Power of Topography to the Topography of Power. A Discourse on Strange Ruralities", Paul C. y Little J, editores, Contested Countryside Cultures Otherness, Marginalization and Rurality, Londres: Routledge. 1997

NAVARRETE, E Adolescentes y trabajo en tres regiones del Estado de México. México: El Colegio Mexiquense, 1992.

PACHECO, L. Los últimos guardianes. Jóvenes rurales e indígenas. Reguillo, R. (coord.). Los Jóvenes en México. Fondo de Cultura Económica, 2010

RAFFESTIN, C. Pour une géographie du pouvoir París: Librairies Techniques, 1980

REDFIELD, Robert (1944) Yucatán una cultura de transición Fondo de Cultura Económica, México, D. F.

REGUILLO, R. Presentación Reguillo, Rossana (coord.). Los Jóvenes en México. Fondo de Cultura Económica, 2010.

STERN, C. Sexualidad juvenil. Más allá del estado del arte, Padilla, J. (coord.), La construcción de lo juvenil. Reunión Nacional de Investigadores sobre Juventud México: Causa Joven.1998

URTEAGA, M. Género, clase y etnia. Los modos de ser joven Reguillo, R. (coord.). Los Jóvenes en México. Fondo de Cultura Económica, 2010

WIRTH L. El urbanismo como modo de vida Ediciones 3 Buenos Aires (1962)

WOODS, M. Rural Geography: Blurring Boundaries and Making Connections, Progress in Human Geography, vol. 33, núm. 6, diciembre, Londres: Sage, pp. 849-858, 2009. 